\title{
Selective Quasienergies from Short Time Cross-Correlation Probability Amplitudes by the Filter-Diagonalization Method
}

\author{
Markus Glück $^{(a)}$, H. Jürgen Korsch ${ }^{(a)}$ and Nimrod Moiseyev ${ }^{(b)}$ \\ (a) Fachbereich Physik, Universität Kaiserslautern, D-67653 Kaiserslautern, Germany \\ ${ }^{(b)}$ Department of Chemistry and Minerva Center for Non Linear Physics of Complex Systems, \\ Technion - Israel Institute of Technology Haifa 32000, Israel
}

\begin{abstract}
The Filter-Diagonalization Method is applied to time periodic Hamiltonians and used to find selectively the regular and chaotic quasienergies of a driven $2 \mathrm{D}$ rotor. The use of $N$ cross-correlation probability amplitudes enables a selective calculation of the quasienergies from short time propagation to the time $T^{(N)}$. Compared to the propagation time $T^{(1)}$ which is required for resolving the quasienergy spectrum with the same accuracy from auto-correlation calculations, the cross-correlation time $T^{(N)}$ is shorter by the factor $N$, that is $T^{(1)}=N T^{(N)}$.
\end{abstract}




\section{INTRODUCTION}

Filter diagonalization was recently introduced by Neuhauser [1] and Neuhauser and Wall [2-4] as a general method to extract frequencies (poles) from a given signal. The approach has been extensively used in quantum dynamics and general contexts, by several groups [5-7]. In the calculation of photoabsorption probabilities filter diagonalization together with the $\left(t, t^{\prime}\right)$ method has been used as an effective tool to extract quasienergies from the autocorrelation function without full-matrix diagonalization [8].

Recently it has been proposed to extract the energy spectrum of the studied system from the cross-correlation functions rather than from the auto-correlation amplitudes $[1,9]$. This approach enables us [9] to extract the resonance positions and widths (associated with the complex eigenvalues of the complex scaled Hamiltonian) from very short time propagations which are too short to get the spectrum from auto-correlation calculations.

Mandelshtam and Taylor's simple box filter [5] was found to be very efficient and we will also use it in the present application of the filter diagonalization method with crosscorrelation functions to time periodic Hamiltonians. In particular, it will be shown that:

(a) The filter diagonalization method is applicable to time periodic Hamiltonians, $H(t)=$ $H(t+T)$, provided the auto-correlation and the cross-correlation amplitudes are calculated or measured at $t=T, 2 T, 3 T, \ldots$ and not at any other time interval.

(b) The quasienergy (QE) spectrum can be extracted either from the auto-correlation or from the cross-correlation amplitudes.

(c) The QE which are predominantly populated by the initial state(s) are most accurately obtained. When the initial states are localized in the chaotic regime of the classical phase space all chaotic QE states are obtained, whereas regular QE states are obtained when the initial states are localized in the regular part of the stroboscopic Poincaré surface of section. 
(d) Accurate QE spectra can be obtained from $N$ cross-correlation functions propagated from $t=0$ to $t=m T$, whereas $N$ times more propagation steps (i.e. up to $t=$ $m N T$ ) are required in order to obtain an accurate spectrum from $N$ auto-correlation amplitudes.

This allows to replace one long time by few short time calculations which is a useful tool in systems for which the numerical effort of long time calculations increases with time or for which only the short time regime yields significant information, e.g. in the calculation of fast decaying resonances [9].

\section{FILTER DIAGONALIZATION FOR TIME PERIODIC HAMILTONIANS}

The filter diagonalization method enables one to calculate seletively the energy spectrum of time independent systems from the auto-correlation amplitudes

$$
C_{n}(t)=\left\langle\phi_{n}|\hat{U}(t)| \phi_{n}\right\rangle
$$

or from the cross-correlation amplitudes

$$
C_{n, m}(t)=\left\langle\phi_{n}|\hat{U}(t)| \phi_{m}\right\rangle
$$

where $\hat{U}(t)$ is the time evolution operator, $n, m \in\{1,2, \ldots, N\}[9]$ and $t<\mathcal{T}$. The key point is that the time $\mathcal{T}$ is too short to get the spectrum by FFT or by other known methods [1-5]. The energy eigenvalues of the system, $E$, are obtained by solving a generalized eigenvalue problem,

$$
\mathbf{U} \vec{\Psi}=\lambda \mathbf{S} \vec{\Psi}
$$

where $\mathbf{U}=\mathbf{U}(\Delta t)$ is the time propagator for a time step $\Delta t$ and $\mathbf{S}$ is the overlap matrix of the filter basis functions. Both matrices consist of $N \times N$ submatrices with the dimension $N_{f} \times N_{f}$ where $N$ is the number of initial states for the cross-correlation amplitudes and $N_{f}$ the number of filter basis functions (for further details see [9]). We should emphasize that 
the only input data needed to calculate $\mathbf{U}$ and $\mathbf{S}$ are the $C_{n}(t)$ or $C_{n, m}(t)$ at the discrete times $t=k \Delta t, k=0,1,2, \ldots, N_{t}$ where $N_{t} \Delta t=\mathcal{T}$. There is no need to know even for which Hamiltonian system the $C_{n}(t)$ ( or the $C_{n, m}(t)$ ) were calculated or measured. The eigenvalues and the energy spectrum are related by $E=i \hbar(\Delta t)^{-1} \ln (\lambda)[9]$ (note that the quasienergies are defined up to multiples of $\hbar \omega)$.

The derivation of Eq. (3) for time independent systems is based on two facts:

(I) The energy eigenstates $\left|\Phi_{E}\right\rangle$ of the Hamiltonian $\hat{H}$ are also eigenstates of the time evolution operator $\hat{U}(t)=\exp (-i \hat{H} t / \hbar)$,

$$
\hat{U}(t)\left|\Phi_{E}\right\rangle=\exp (-i \hat{E} t / \hbar)\left|\Phi_{E}\right\rangle
$$

(II) The filter basis functions, which are taken as a basis set to diagonalize the time evolution operator $\hat{U}$, are defined as

$$
\left|\Phi_{E}\right\rangle=\frac{1}{\mathcal{T}} \int_{0}^{\mathcal{T}} \mathrm{d} t \mathrm{e}^{\mathrm{i} E t / \hbar} \hat{U}(t)|\phi(0)\rangle
$$

In the limit $\mathcal{T} \rightarrow \infty$ they are eigenstates if $E$ is an eigenvalue.

For time dependent systems the first condition is, of course, not fulfilled since the time evolution operator is not $\exp (-i \hat{H}(t) t / \hbar)$. We can overcome this difficulty by expressing the time evolution operator by the $\left(t, t^{\prime}\right)$ method [10]. It implies that the Hamiltonian $\hat{H}$ in the first conditions and in the expressions used to derive Eq. (3) (see [9]) should be replaced by the Floquet or quasienergy operator $\hat{\mathcal{H}}=\hat{p}_{t^{\prime}}+\hat{H}\left(t^{\prime}\right)$ which is time (i.e. $\left.t\right)$ independent. Here $t^{\prime}$ acts as an additional coordinate and not as a parameter. The momentum operator $\hat{p}_{t^{\prime}}$ is defined as usual. Using the $\left(t, t^{\prime}\right)$, we can get an analytical form of the time evolution operator,

$$
\hat{U}(t)=\int \mathrm{d} t^{\prime} \hat{\delta}\left(t-t^{\prime}\right) \exp \left(-\mathrm{i} \hat{\mathcal{H}}\left(t^{\prime}\right) t / \hbar\right)
$$

This fact enables the derivation of time independent scattering theory for time dependent Hamiltonians [10,11]. Let us now check if the above mentioned conditions are fulfilled for time periodic systems: 
(I) For time periodic Hamiltonians the quasienergy solutions $\left|\Psi_{E}\right\rangle$ (which are defined as eigenstates of the Floquet operator) are given by

$$
\hat{\mathcal{H}}(t)\left|\Psi_{E}(t)\right\rangle=E\left|\Psi_{E}(t)\right\rangle
$$

with the time periodic functions

$$
\left|\Psi_{E}(t)\right\rangle=\left|\Psi_{E}(t+T)\right\rangle
$$

On the other hand, using equation (6)

$$
\hat{U}(t)\left|\Psi_{E}(0)\right\rangle=e^{-\mathrm{i} E t / \hbar}\left|\Psi_{E}(t)\right\rangle
$$

so that condition (I) (see eqn. (4)) is fulfilled if $\left|\Psi_{E}(0)\right\rangle=\left|\Psi_{E}(t)\right\rangle$ which is only the case for $t=n T$ (see Eq. (8)).

(II) As condition (I) is only fulfilled at the discrete times $t=n T, n=0,1,2, \ldots$, the integration in the definition of the filter basis functions (Eq. 5) has to be replaced by a summation. So, for time periodic Hamiltonians the filter basis functions are given by

$$
\left|\Psi_{E}\right\rangle=\frac{1}{N_{t}+1} \sum_{n=0}^{N_{t}} \mathrm{e}^{\mathrm{i} n E T / \hbar} \hat{U}(n T)|\phi(0)\rangle
$$

Using this definition we see that in the limit $N_{t} \rightarrow \infty$ the filter basis function $\Psi_{E}$ is an eigenfunction of the Floquet operator if $E$ is a quasienergy and condition (II) is fulfilled.

This shows that in the case of time periodic Hamiltonians we have to take $\Delta t=T$ and to modify the definition of the filter basis functions. Only then the QE spectrum can be obtained by the filter diagonalization method. This conclusion has been confirmed by our numerical calculations. 


\section{SELECTIVE QUASIENERGIES FROM SHORT TIME CROSS-CORRELATIONS}

Let us take as an illustrative numerical example a 2D driven rotor Hamiltonian

$$
\hat{H}(t)=\frac{\hat{p}_{\phi}^{2}}{2}+\cos (\phi) \cos (\omega t)
$$

modeling, e.g, a dipole in an oscillating field (the rotational momentum $\hat{p}_{\phi}$ is $\hbar m$, where $m \in$ $\{0, \pm 1, \pm 2, \ldots\}$ is the rotational quantum number). This system, also known as the double resonance model, is one of the models investigated extensively in context with quantum chaos (see, e.g., [12-17]).

The classical dynamics of the system (11) is chaotic, i.e. it shows a mixture of regular and chaotic dynamics. Stroboscopic Poincaré sections of the classical phase space at $t=n T$, $(n=0,1,2, \ldots)$ are shown in Fig. 1 for the frequencies $\omega=0.6$ and $\omega=1.2$. For $\omega=0.6$ we have a clear division of phase space into a single inner chaotic and two outer regular regions. For $\omega=1.2$ two inner (classically disconnected) regular islands appear centered at the two stable resonant co- and counter-rotating periodic modes of the rotor.

In the quantum studies, it has been found that in the semiclassical regime the quasienergy states can be divided into two classes of eigenstates [15,17]:

One class of solutions are extended quasienergy states which populate almost uniformly the free rotor states below a specific value of $|m|$ in $2 \mathrm{D}$ problems and below the rotational quantum number $j$ in the 3D case (see Figs. 7,17,18 in [15] and Fig. 10.a in [16]). The strong random population of all contributing free rotor basis functions is reflected in the Husimi distribution which shows almost equal probability in the classically chaotic region (see Fig. 10b in [16]). The states which which belong to this class of solutions are referred to as chaotic quasienergy states.

The second class of solutions which are referred to as regular states, are localized both in the free rotor basis set and in the stroboscopic Poincare surface of sections. See Fig. 6 in [16] for states the Husimi distribution of which is localized in the inner regular islands of the chaotic "sea" and Fig. 9 in [16] for states which are localized along outer regular orbits. 
The quasienergies for $\hbar=0.1$ and $\omega=0.6$ and also for $\omega=1.2$ were calculated using 81 free rotor states as a basis set. The numerically exact quasienergies (chaotic and regular following the definitions given above) were obtained from the diagonalization of the Floquet time evolution operator $\hat{U}(T)$. A number of 40 of these states could be classified as chaotic in agreement with the estimate from the area of the chaotic region in phase space. The quasienergies of chaotic states for $\omega=0.6$ in the representative interval $0.01<E<0.02$ are given in the second column of Table I.

To demonstrate the selectivity of the filter diagonalization method, we first compute only the chaotic quasienergy states. As the chaotic quasienergy states populate almost uniformly the free rotor states, in reverse the free rotor states populate almost uniformly the chaotic QE states. So, we expect to populate all chaotic states using as initial states the $n=0,1,2,3,4$ free rotor states. We calculated the spectrum from the auto-correlation functions, $C_{n}(t)$ with $n=0,1,2,3,4$, and also from the cross-correlation amplitudes, $C_{n, m}(t)$ where $n, m=0,1,2,3,4$.

For the first calculation the Neuhauser method [2] with Mandelshtam-Taylor filter was used. The recent generalization of the filter method by Narevicius, Korsch, Neuhauser and Moiseyev [9] was used to extract the quasienergy spectrum from the cross-correlation functions. The results for $\omega=0.6$ are presented in Table I.

The first lines in boxes of the third column are the chaotic quasienergies obtained from long time propagated auto-correlation functions $C_{n}(t)$ where $t=0, T, 2 T, \ldots, 100 T$. The reason for sampling the auto-correlation functions every time period has been explained above. The results obtained from short time propagated auto-correlation functions with $t=0, T, 2 T, \ldots, 20 T$ are presented in the third (last) lines of the boxes in the third column of Table I.

The deviation of the quasienergies obtained by the filter diagonalization method from the exact values are shown in the last column in Table I. The results obtained from long time $(100 T)$ propagations are in a remarkable agreement with the exact values. Note that the Fourier transform of the same functions provide results, which are by several order of 
magnitudes less accurate.

The results obtained from the short time (20T) propagation are bad. In some cases it was impossible to allocate a spectral line from the analysis of the auto-correlation functions to the exact $\mathrm{QE}$ value. In the best case short time results were less accurate by more than four orders of magnitude from the the long time result.

The imaginary part of the QE should be equal to zero as the QE states are bounded states, so we use the imaginary part of the calculated QE (fourth column in Table I) as an independent error estimate of the filter diagonalization. Another error estimate can be obtained from the variations of the spectral lines (fifth column in Table I) as the time evolution operator was diagonalized by the filter method at $t=T, 2 T$ and $3 T$ (for more detailed explanation see [5] and [9]). In comparison of the last three columns the independent errors are about equal to the actual error of the results.

It is interesting to compare the errors of the long time auto-correlation results with the the population of the quasienergy states by the initial states ( first column in Table I). The error of $3.38 \cdot 10^{-9}$ was obtained for the state which has been populated in the probability of 0.000474 where the error of $5.39 \cdot 10^{-11}$ was obtained for a state that was populated in higher probability of 0.0819 . Another interesting results that will be discussed later in more details is that the largest errors are obtained for almost degenerate states.

The rational behind the success of the filter diagonalization method to extract the QE spectrum from the long time propagated auto-correlation functions and its failure to do so for the short time propagation is as follows:

Each one of the QE state fills up a specific region in the classical phase space. The minimal time which is required to extract the $\mathrm{QE}$ value from $C_{n}(t)$ is the time which it takes for the $n$-th initial state to cover entirely this specific region in the classical phase space (this can be seen by carrying out time dependent Husimi distribution calculations). Our results clearly show that $20 T$ is not enough to do so but $100 T$ is more than enough.

Following this rational we expect that in the case of $N \times N$ cross-correlation calculations the $N$ initial states together will cover the entire bounded chaotic classical phase space 
within $100 / N$ periods! That is, for $N=5$ it is enough to calculate (or to measure) $C_{n, m}$ with $n, m=0,1,2,3,4$ up to $t=20 T$. The results of this calculation are presented in the second lines of the boxes in the third column in Table I. The results are in excellent agreement with the exact $\mathrm{QE}$ values and by far better than the results obtained from the short time (also 20T) propagations of the five separated auto-correlation functions.

This is observed for all chaotic QE solutions in Fig. 2, where the errors in the QE are shown vs. the computed QE values. The results obtained from the short time propagated cross-correlations data are more accurate in several orders of magnitude (on the average by 4) than the results computed from the short time propagated auto-correlations amplitudes.

In order to show that indeed all chaotic QE were obtained by the filter diagonalization method using five short time (20T) propagated cross-correlation functions we plotted in the upper part of Fig. 3 the exact $\mathrm{QE}$ values and their summed population by the five initial states, whereas in the lower part of Fig. 3 the QE spectrum obtained by the filter diagonalization and the corresponding values of the inverse of the error are plotted. In Fig. 4 we show for comparison the same plot for the results obtained by the filter diagonalization method from the short time propagated auto-correlation functions (the long time autocorrelation functions provide good results as in Fig. 3). Unlike the results presented in Fig. 4, the QE spectrum obtained from the short time propagated 5-cross-correlation functions (Fig. 3) is very accurate. In the scale of the plot of Fig. 3, all of the QE are not only indistinguishable from the exact values but also the small errors are proportional to the inverse of the populations of the $\mathrm{QE}$ by the initial states. That is, as the populations is larger the error is smaller.

Following the rational behind the success to filter out the QE spectrum from short time propagations of cross-correlation functions one may expect that this will be the most efficient procedure for calculating a regular QE since the QE state is localized in a relatively small region of the stroboscopic Poincare surface of section. Indeed this is the situation. However there are cases as shown in Fig. 1b that because of the symmetrical pair of regular islands the regular part of the spectrum (associated with the regular QE states) is almost degenerate. 
The results presented in Table II clearly show that short time propagation calculations with 5-cross-correlation functions cannot resolve the splitting. To get better results one has to increase the time interval, e.g. for $t=100 T$ the agreement is much better (see the last column of Table II). It is interesting to compare the propagation time with the "tunneling time" $\tau=\pi \hbar / \Delta( \pm)$ (second column in Table II), where $\Delta( \pm)$ stands for the energy splitting. We find that Filter Diagonalization with cross-correlation amplitudes can resolve splittings even if the propagation time is remarkably shorter than the tunneling time.

\section{CONCLUDING REMARKS}

Using the filter diagonalization method when the input data are time dependent crosscorrelation functions sampled at $t=T, 2 T, \ldots$ highly accurate quasienergy spectra of time periodic systems can be obtained. The error is smaller for the QE that are more populated by the initial states and larger for almost degenerate states. As a rule of thumb we may conclude that as more initial states are used to calculate the cross-correlation amplitudes than one can filter out the accurate QE spectrum from shorter time propagation calculations.

\section{ACKNOWLEDGMENTS}

This work was done while one of the authors (N.M.) was staying at the University of Kaiserslautern as an Humboldt awardee. The US-Israel Binational Foundation and the Foundation of Promotion of Research at the Technion are acknowledged for partial support. This research has been supported by the Deutsche Forschungsgemeinschaft (SPP 'Zeitabhängige Phänomene und Methoden in Quantensystemen der Physik und Chemie'). 


\section{REFERENCES}

[1] D. Neuhauser, J. Chem. Phys. 93, 2611 (1990)

[2] M.R. Wall and D. Neuhauser, J. Chem Phys. 102, 8011 (1995).

[3] G.-J Kroes, M.R. Wall, J.W. Pang, and D. Neuhauser, J. Chem Phys. 106, 1800 (1997).

[4] J.W. Pang and D. Neuhauser, Chem. Phys. Lett. 252, 173 (1996).

[5] V.A Mandelshtam and H.S. Taylor, J. Chem. Phys. 106, 5085 (1997); Phys. Rev. Lett. 78, 3274 (1997); J. Chem. Phys., submitted.

[6] R. Q. Chen and H. Guo, J. Chem. Phys. 105, 1311 (1996).

[7] H. G. Yu and S. C. Smith, Berich. Bund. Ges. Phys. Chem. 101, 400 (1997).

[8] J. W. Pang, D. Neuhauser and N. Moiseyev, J. Chem. Phys. 106, 6839 (1997).

[9] E. Narevicius, H. J. Korsch, D. Neuhauser and N. Moiseyev, Chem. Phys. Lett. (in press).

[10] U. Peskin and N. Moiseyev, J. Chem. Phys. 99, 4590 (1993).

[11] For a review see: N. Moiseyev, Comments on Atomic and Molecular Physics 31, 87 (1995), and references therein.

[12] G. B. Berman, G. M. Zaslavsky, and A. R. Kolovsky, Sov. Phys.JETP 54, 272 (1981).

[13] A. R. Kolovsky, Phys. Lett. A 157, 474 (1991).

[14] A. R. Kolovsky, in: P. Garbaczewski, M. Wolf, and A. Weron, editors, Chaos - The interplay between stochastic and deterministic behaviour. (Springer, Berlin, Heidelberg, New York, 1995).

[15] N. Moiseyev, H.J. Korsch and B. Mirbach, Z. Phys. D 29, 125 (1994).

[16] V. Averbukh, N. Moiseyev, B. Mirbach and H.J. Korsch, Z. Phys. D 35, 247 (1995). 
[17] T. Gorin, H. J. Korsch, and B. Mirbach, Chem. Phys. 217, 147 (1997). 


\section{TABLES}

TABLE I. Numerically exact quasienergies and results obtained by the filter diagonalization method using long time auto-correlation (first rows, 100 periods), short time cross-correlation (second rows, 20 periods) and the short time auto-correlation amplitudes (third rows, 20 periods) as input data. $\Delta(E)$ denotes the deviations from the numerically exact values. $\operatorname{Im}(E)$ is the imaginary part and Err is the error estimate obtained in the filter diagonalization procedure (see the text). Pop is the summed population probability of the QE state by the initial states used in the propagation.

\begin{tabular}{|c|c|c|c|c|c|}
\hline Pop & $E($ exact $)$ & $E$ (filter) & $\operatorname{Im}(E)$ & Err & $\Delta E$ \\
\hline \multirow[t]{3}{*}{$1.51 \mathrm{e}-01$} & 0.0106163793942 & 0.0106163792312 & $-3.07 \mathrm{e}-10$ & $6.84 \mathrm{e}-10$ & $1.63 \mathrm{e}-10$ \\
\hline & & 0.0106164127588 & $-2.24 \mathrm{e}-08$ & $8.07 \mathrm{e}-08$ & $3.34 \mathrm{e}-08$ \\
\hline & & none & - & - & - \\
\hline \multirow[t]{3}{*}{$1.87 \mathrm{e}-01$} & 0.0111773758931 & 0.0111773759127 & $2.59 \mathrm{e}-11$ & $1.69 \mathrm{e}-10$ & $1.96 \mathrm{e}-11$ \\
\hline & & 0.0111773125827 & $1.67 \mathrm{e}-07$ & $3.56 \mathrm{e}-07$ & $6.33 \mathrm{e}-08$ \\
\hline & & 0.0114161960781 & $-7.72 \mathrm{e}-04$ & $2.76 \mathrm{e}-04$ & $2.39 \mathrm{e}-04$ \\
\hline \multirow[t]{3}{*}{$9.49 \mathrm{e}-02$} & 0.0135287439410 & 0.0135287508368 & $-3.12 \mathrm{e}-09$ & $1.18 \mathrm{e}-09$ & $6.90 \mathrm{e}-09$ \\
\hline & & 0.0135289043188 & $2.59 \mathrm{e}-09$ & $3.11 \mathrm{e}-07$ & $1.60 \mathrm{e}-07$ \\
\hline & & none & - & - & - \\
\hline \multirow[t]{3}{*}{$4.74 \mathrm{e}-04$} & 0.0136237870670 & 0.0136237904429 & $-4.83 e-09$ & $1.11 \mathrm{e}-08$ & $3.38 \mathrm{e}-09$ \\
\hline & & 0.0136237964034 & $-2.60 \mathrm{e}-07$ & $5.02 \mathrm{e}-07$ & $9.34 \mathrm{e}-09$ \\
\hline & & none & - & - & - \\
\hline \multirow[t]{3}{*}{$4.20 \mathrm{e}-02$} & 0.0136605804640 & 0.0136605575681 & $-1.35 \mathrm{e}-08$ & $5.42 \mathrm{e}-08$ & $2.29 \mathrm{e}-08$ \\
\hline & & 0.0136608645320 & $1.40 \mathrm{e}-07$ & $5.74 \mathrm{e}-07$ & $2.84 \mathrm{e}-07$ \\
\hline & & 0.0138393729925 & $4.67 \mathrm{e}-03$ & $5.99 \mathrm{e}-03$ & $1.79 \mathrm{e}-04$ \\
\hline \multirow[t]{3}{*}{$2.04 \mathrm{e}-01$} & 0.0141612213828 & 0.0141612216830 & $3.75 \mathrm{e}-10$ & $1.08 \mathrm{e}-09$ & $3.00 \mathrm{e}-10$ \\
\hline & & 0.0141612380743 & $6.92 \mathrm{e}-09$ & $3.54 \mathrm{e}-08$ & $1.67 \mathrm{e}-08$ \\
\hline & & 0.0145396441221 & $-5.65 \mathrm{e}-05$ & $1.21 \mathrm{e}-04$ & $3.78 \mathrm{e}-04$ \\
\hline \multirow[t]{3}{*}{$8.19 \mathrm{e}-02$} & 0.0169683947620 & 0.0169683948159 & $3.16 \mathrm{e}-11$ & $3.56 \mathrm{e}-10$ & $5.39 \mathrm{e}-11$ \\
\hline & & 0.0169683903456 & $-7.97 \mathrm{e}-09$ & $1.65 \mathrm{e}-08$ & $4.42 \mathrm{e}-09$ \\
\hline & & 0.0176839217544 & $6.29 \mathrm{e}-04$ & $3.05 \mathrm{e}-04$ & $7.16 \mathrm{e}-04$ \\
\hline \multirow[t]{3}{*}{$2.43 \mathrm{e}-01$} & 0.0189157686746 & 0.0189157694578 & $1.56 \mathrm{e}-11$ & $3.36 \mathrm{e}-10$ & $7.83 \mathrm{e}-10$ \\
\hline & & 0.0189157485962 & $5.19 \mathrm{e}-08$ & $1.13 \mathrm{e}-07$ & $2.01 \mathrm{e}-08$ \\
\hline & & 0.0187453836203 & $8.93 \mathrm{e}-05$ & $1.17 \mathrm{e}-04$ & $1.70 \mathrm{e}-04$ \\
\hline \multirow[t]{3}{*}{$1.39 \mathrm{e}-01$} & 0.0195380171655 & 0.0195380181074 & $-1.07 \mathrm{e}-10$ & $8.94 \mathrm{e}-10$ & $9.42 \mathrm{e}-10$ \\
\hline & & 0.0195378884673 & $9.43 e-08$ & $3.19 \mathrm{e}-07$ & $1.29 \mathrm{e}-07$ \\
\hline & & 0.0195500567555 & $-4.91 \mathrm{e}-04$ & $7.23 \mathrm{e}-04$ & $1.20 \mathrm{e}-05$ \\
\hline
\end{tabular}


TABLE II. Five pairs of almost degenerate regular quasienergies and results obtained by the filter diagonalization method when the long time (100 periods) and short time (20 periods) propagated cross-correlation amplitudes are used as input data. $\Delta( \pm)$ stands for the energy splitting between any two almost degenerate QE states, $\tau$ denotes the tunneling time $\tau=\pi \hbar / \Delta( \pm)$.

\begin{tabular}{lcccc}
\hline \hline$\Delta( \pm)$ & $\tau / T$ & $E($ exact $)$ & $E(20 T)$ & $E(100 T)$ \\
\hline $3.55 \mathrm{e}-9$ & $1.69 \mathrm{e}+7$ & 0.02723262310 & 0.02723262310 & \\
& & 0.02723262665 & & 0.02723262608 \\
\hline $2.49 \mathrm{e}-7$ & $2.41 \mathrm{e}+5$ & -0.02273547656 & -0.02273559570 & -0.02273560166 \\
& & -0.02273572571 & & -0.02274478376 \\
\hline $1.54 \mathrm{e}-5$ & $3.90 \mathrm{e}+3$ & 0.04585922247 & 0.04586688280 & 0.04585965574 \\
& & 0.04587453432 & & 0.04587501585 \\
\hline $7.73 \mathrm{e}-4$ & $7.75 \mathrm{e}+1$ & -0.00657529061 & -0.00684298947 & -0.00657528341 \\
& & -0.00735152721 & & -0.00735153332 \\
\hline $1.03 \mathrm{e}-3$ & $5.83 \mathrm{e}+1$ & 0.05770679131 & 0.05764687657 & 0.05770679116 \\
& & 0.05873970381 & 0.05870356560 & 0.05873970389 \\
\hline \hline
\end{tabular}




\section{FIGURES}
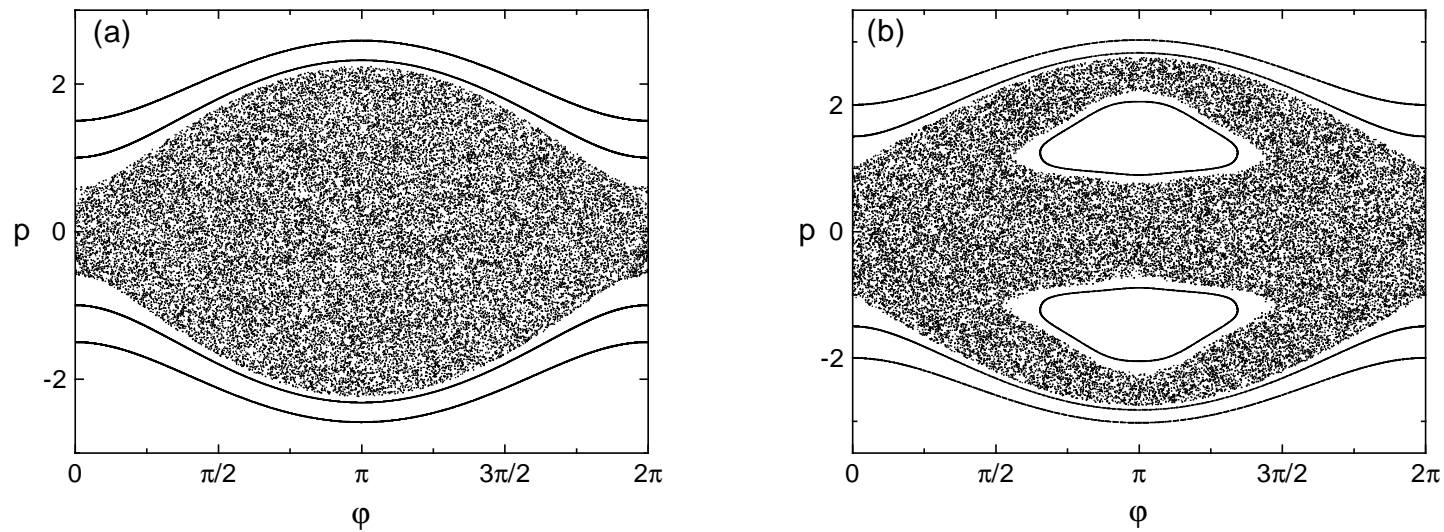

FIG. 1. Stroboscopic Poincare section of classical phase space at $t=n T,(n=0,1,2, \ldots)$. All points in the chaotic region result from one single classical trajectory: (a) driven rotor with the frequency $\omega=0.6$, (b) driven rotor with the frequency $\omega=1.2$.

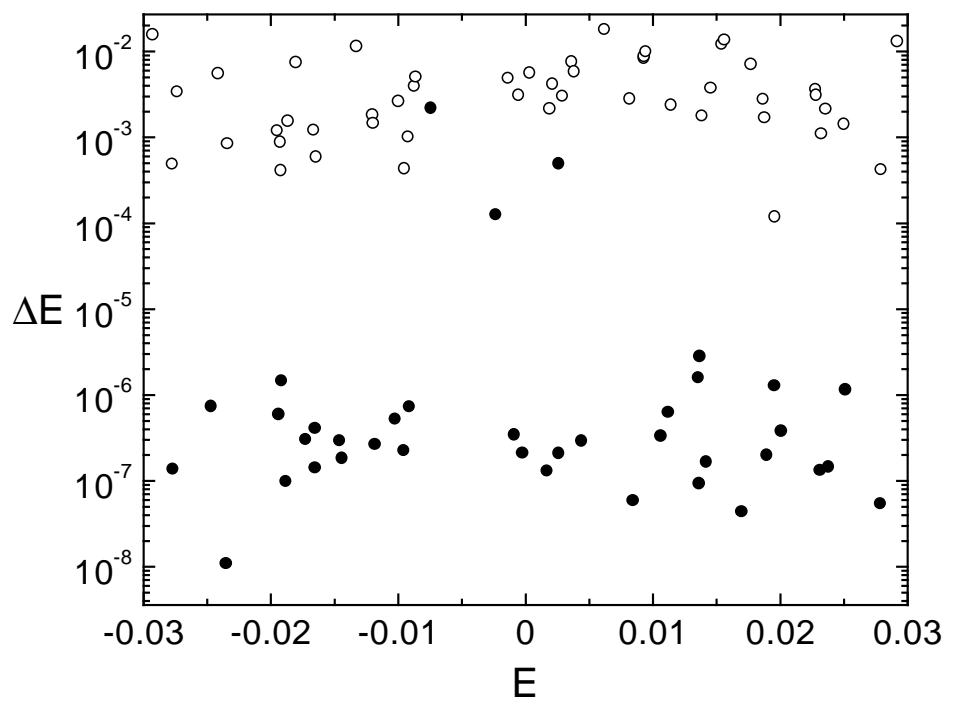

FIG. 2. The deviation of the quasienergies obtained by the filter diagonalization method from the excact numerical values. The open circles (large error) were obtained by filtering out the spectrum from the short time (20 periods) propagation of five different auto-correlation amplitudes. The full circles (small error) were obtained from 5-cross-correlation function amplitudes. 


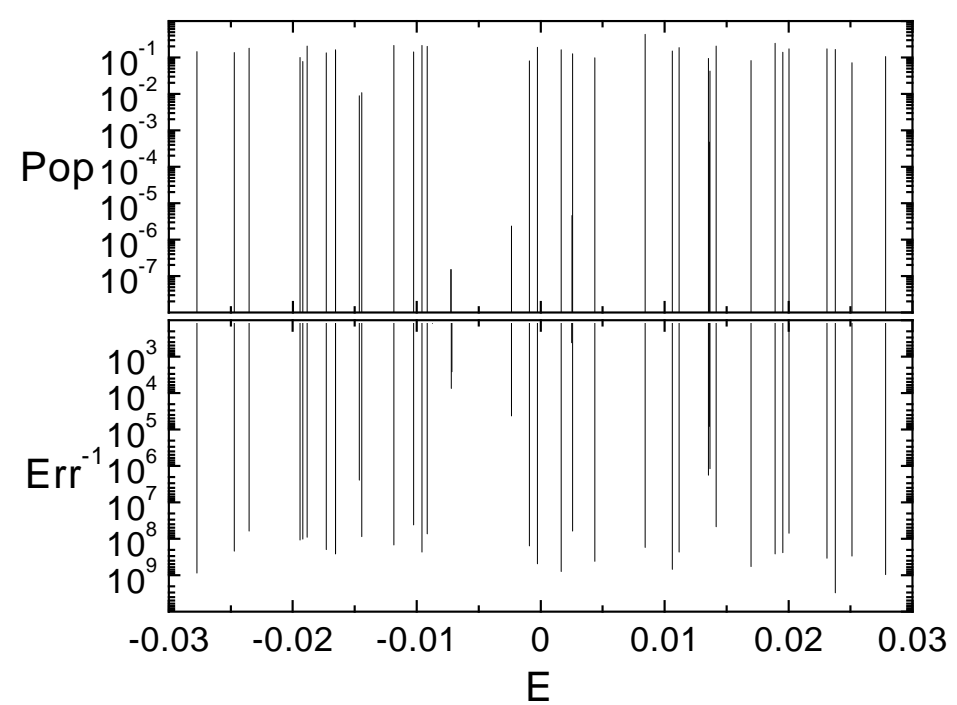

FIG. 3. The upper plot shows the numerically exact quasienergies and the summed population probabilities of the initial states used in the time propagation calculations. The lower plot shows the QE spectrum obtained by the filter diagonalization calculations using the short time propagated 5 cross-correlation amplitudes versus the inverse of the calculated error estimate. Both spectra show excellent agreement.

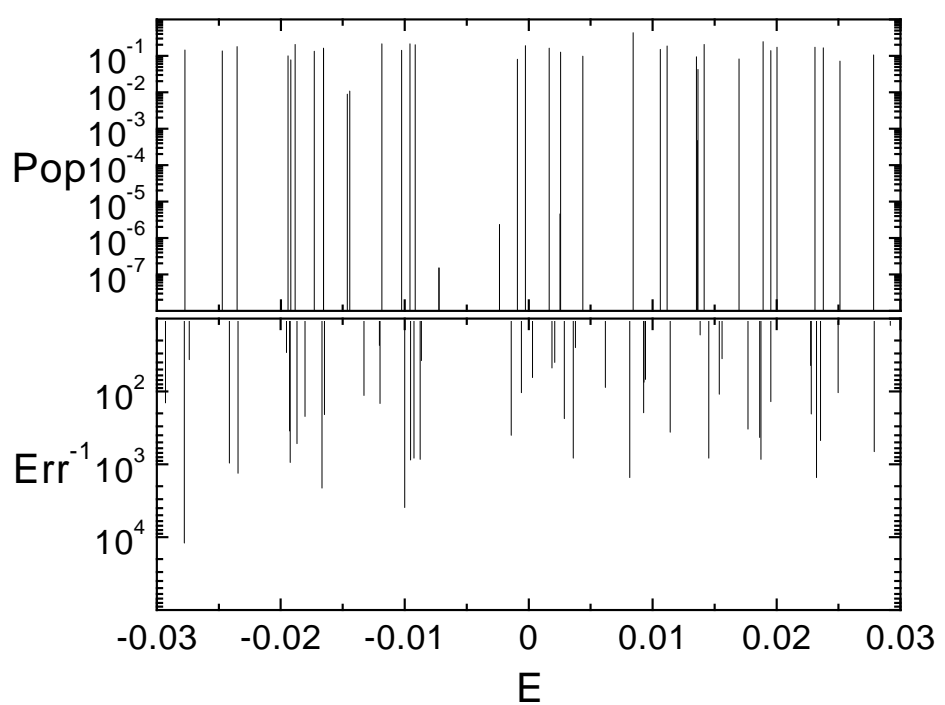


FIG. 4. As in Fig. (3), but five separated short time propagated auto-correlation amplitudes were used as an input data in the filter diagonalization method. The agreement of the spectra is bad. 